\title{
Manejo forestal sostenible del bosque y monitoreo ecológico en dos bosques muy húmedos tropicales de Zona Norte de Costa Rica
}

\section{Sustainable forest management of the forest and ecological monitoring in two very humid tropical forests of the Northern Zone of Costa Rica}

Cynthia Salas-Garita $\bullet$ Gabriela Jones-Román²

\begin{abstract}
An ecological monitoring was carried out on two properties subject to sustainable forest management (SFM), located in the Northern Zone of Costa Rica. For monitoring purposes, the basal area was chosen as the gross filter indicator, and the composition and diversity of diurnal butterflies of the Nymphalidae, Papilionidae and Pieridae families was chosen as a fine filter indicator. The results indicate that forests subjected to SFM, which were also affected by the passage of Hurricane Otto, suffered a significant loss of basal area, which implies adjustments in the new cutting cycle to ensure the recovery of forest areas. It was found that as lower forest cover, the greater abundance, richness and composition of butterflies. In order to use the monitoring of butterflies as an indicator of sustainability, a greater effort is required to achieve the construction of reference lines for this indicator.
\end{abstract}

Key words: Butterflies, basal area, indicator, reference lines, thresholds of change.

1. Escuela de Ingeniería Forestal, Tecnológico de Costa Rica (Doctorante del programa Ciencias Naturales para el Desarrollo (DOCINADE), énfasis en Recursos Naturales); Cartago, Costa Rica; cysalas@tec.ac.cr 


\section{Resumen}

Se realizó un monitoreo ecológico en dos propiedades sometidas a manejo forestal sostenible (MFS), ubicadas en la Zona Norte de Costa Rica. Para el monitoreo se eligió como indicador de filtro grueso el área basal y como indicador de filtro fino la composición, y diversidad de mariposas diurnas de las familias Nymphalidae, Papilionidae y Pieridae. Los resultados indican que los bosques sometidos a MFS, que además fueron afectadas por el paso del Huracán Otto, sufrieron una pérdida importante de área basal, lo que implica ajustes en el nuevo ciclo de corta que aseguren la recuperación de las áreas de bosque. Se comprobó que a menor cobertura forestal mayor abundancia, riqueza y composición de mariposas. Para lograr utilizar los monitoreos de mariposas como indicador de la sostenibilidad se requiere de un esfuerzo mayor para lograr construir líneas de referencia para este indicador.

Key words: Mariposas, área basal indicador, líneas de referencia, umbrales de cambio.

\section{Introducción}

El manejo forestal sostenible del bosque (MFS) es todo un reto porque supone utilizar los recursos a través de la gestión misma del bosque, de tal forma que la intensidad de su utilización no vaya en detrimento de la capacidad futura de producción, que conserve la biodiversidad, su capacidad de regeneración, sus funciones ecológicas y que además siga cumpliendo un papel fundamental en la economía y la sociedad [1], [2].

La cobertura forestal en Costa Rica asciende a un 52,4 $\%$ y la mayor proporción la tiene el bosque maduro que representa el $31 \%$ del país [3]. En este contexto, el MFS del bosque es permitido cuando existe un Plan de Manejo Forestal que cumple con Principios, Criterios e Indicadores de Sostenibilidad que sirven de base para la elaboración de ese Plan de Manejo [4], siendo el Área de Conservación Arenal Huetar Norte, la región del país donde se registra la mayor cantidad de planes de manejo aprobados [5].

El MFS en Costa Rica se ha dirigido especialmente al aprovechamiento del producto madera en ciclos de al menos 15 años, dejando en el intermedio la recuperación del bosque. Sin embargo, el manejo forestal del bosque bajo el paradigma de manejo adaptativo no ha dejado claro quién debe realizar el monitoreo de las áreas bajo manejo y en qué consiste ese monitoreo para el período entre cosechas [1].
El compromiso que hace posible el MFS depende de la voluntad de los interesados en valorar los objetivos del largo y corto plazo, implementando un plan de protección y monitoreo después de la primera cosecha [6]. En Costa Rica el monitoreo sobre áreas de bosque bajo MFS no ha sido masivo; no obstante, se tienen elementos generales de la planificación de la primera cosecha que podrían permitir planificar la segunda, utilizando los registros previos, pero estos, una vez más, se concentran en la valoración del producto madera. Queda entonces la duda de cuál es el monitoreo que se debe realizar sobre otros elementos de biodiversidad y cuáles afectaciones del manejo forestal podrían tener una importancia tal sobre esos otros elementos que requieran de atención. Aunque estas preguntas no tienen respuestas definitivas, sí se reconoce que la biodiversidad en el bosque es de relevancia, por ser la que permite el mantenimiento de los diferentes procesos ecológicos que son necesarios para garantizar, que los bosques sigan produciendo los diferentes bienes y servicios de interés económico.

Además, las alteraciones en el ecosistema podrían causar perturbaciones indeseables sobre la biodiversidad, lo que podría afectar su sostenibilidad [7]. Es por ello que se sugiere que se realicen los monitoreos ecológicos sobre especies indicadoras de perturbación [8], [9] lo que además permitiría evaluar los efectos de la gestión forestal [10]. Ese monitoreo consiste en implementar un seguimiento a especies que son consideradas indicadoras y que están relacionadas con la biodiversidad del bosque [7]. Implementar este tipo de monitoreo permite evaluar el grado de perturbación, de manera indirecta examinando y monitoreando si se están presentando cambios en la composición, estructura y función de los ecosistemas como consecuencia del impacto que producen el aprovechamiento forestal maderable en los bosque naturales [8], [9].

En este sentido, Ordóñez et al. [11] reconocieron que los monitoreos de mariposas podrían ser de utilidad, por ser un grupo sensible al cambio en el hábitat en razón del manejo forestal [10]. Las mariposas diurnas se consideran uno de los grupos más abundantes y diversificados en los bosques tropicales y el estudio de la diversidad y composición de las especies presentes en un ecosistema puede evidenciar, además, situaciones relacionadas con fragmentación de los bosques donde se presume que la diversidad es mayor que en los bosques no perturbados [12]-[14].

Este estudio se presenta con el objetivo de analizar si es factible realizar monitoreos ecológicos con indicadores de filtro grueso como el área basal y de filtro fino como mariposas, en boques con MFS, que permitan determinar si los resultados obtenidos pueden ser de utilidad para 
evaluar la sostenibilidad de los bosques y generar con ellos indicadores útiles para esa evaluación.

\section{Materiales y métodos}

\section{Sitio de Estudio}

El trabajo de campo se realizó en los Cerros de Yucatán, perteneciente al distrito de Pital, cantón de San Carlos, Alajuela, Costa Rica. Las fincas muestreadas se encuentran dentro de los límites del Parque Nacional Maquenque y corresponden con bosques de propiedad privada que han sido manejados según la normativa nacional relacionada con MFS en bosques.

Sitio 1: Tiene un área de 110,5 ha, su último aprovechamiento se ejecutó en la estación seca de los años 2014-2016. Al momento del monitoreo se encontró el sitio en recuperación, no sufrió daños significativos con el paso del Huracán Otto en noviembre del 2016, excepto por la caída de algunos árboles de forma aislada, los cuales no fueron extraídos. Según el plan de manejo aprobado, la extracción promedio de árboles en las áreas productivas asciende a 2,5 árb ha-1 incluyendo los árboles caídos, equivalente a $11,4 \mathrm{~m}^{3} \mathrm{ha}^{-1}$.

Sitio 2: Tiene una extensión de 111,07 ha, y el aprovechamiento más reciente terminó en mayo 2017. Este sitio sufrió daños producidos por el paso del Huracán Otto y la madera caída fue autorizada para extracción. El manejo forestal autorizado permitió la extracción promedio de 1,5 árb ha-1 incluyendo los caídos; no obstante, producto de los efectos del huracán Otto, se autorizó que se suspendiera la corta de árboles y se aprovechara solo lo que huracán Otto afectó, de esta forma de los 105 árboles en pie autorizados, solo se cortaron 34 individuos, el resto se dejaron en pie y se extrajeron adicionalmente 112 árboles caídos por el huracán lo que redujo la extracción a 1,3 árb $\mathrm{ha}^{-1}$, equivalente a 4,8 $\mathrm{m}^{3} \mathrm{ha}^{-1}$. Aunque en apariencia el aprovechamiento es de bajo impacto, el mismo estuvo concentrado en ciertas áreas del bosque donde masivamente se registró caída de árboles por efecto del huracán. Esto provocó una concentración de los claros producidos no tanto por corta sino por efectos del huracán y la extracción autorizada.

Ambos sitios de estudio pertenecen a la Zona de Vida Bosque Muy húmedo Tropical característicos de la zona bajas de la Zona Norte de Costa Rica con menos de $100 \mathrm{msnm}$. En la figura 1, se muestran los límites de las fincas muestreadas.

Estos dos sitios fueron elegidos porque representan condiciones diferenciadas de manejo forestal en su segundo ciclo de corta. En el Sitio 1 se planificó y ejecutó un MFS. En el Sitio 2 se planificó el MFS y se empezó a ejecutar pero los embates del Huracán Otto hicieron que el MFS no se pudiera terminar de ejecutar como se había planeado y finalmente lo que se ejecutó fue un aprovechamiento de madera caída.

\section{Determinación de línea base de área basal y medición actual}

Para tomar decisión sobre línea base de área basal para los árboles con diámetro mayor a $10 \mathrm{~cm}$ se procesó información de parcelas permanentes de muestreo establecidas en el año 1994 en el Sitio 1 y 1995 en el Sitio 2. Además, se analizó la información del muestreo realizado para efectos de la elaboración de los planes de manejo de cada sitio para conocer el área basal de referencia después de 1 ciclo de corta, las mediciones se realizaron en el 2013 para el Sitio 1 y en el 2015 para el Sitio 2.

En cada sitio evaluado se establecieron parcelas temporales de muestreo de $100 \mathrm{~m} \times 10 \mathrm{~m}$ con el fin de determinar el área basal del bosque, para árboles mayores de $10 \mathrm{~cm}$ de diámetro medido a $1,3 \mathrm{~m}$ del suelo (DAP) al momento del muestreo de las mariposas, esto fue después del aprovechamiento del segundo ciclo de corta.

Adicionalmente, se midió temperatura ambiental $\left({ }^{\circ} \mathrm{C}\right)$ y humedad relativa (5) a nivel de piso (a $1 \mathrm{~m}$ del suelo) en 40 puntos de muestreo distribuidos en los transeptos de muestreo de las mariposas, para lo cual se utilizó un Kestrell 3000. En estos mismos puntos de muestreo se hizo la determinación del porcentaje de cobertura forestal utilizando una cámara con lente ojo de pescado [15].

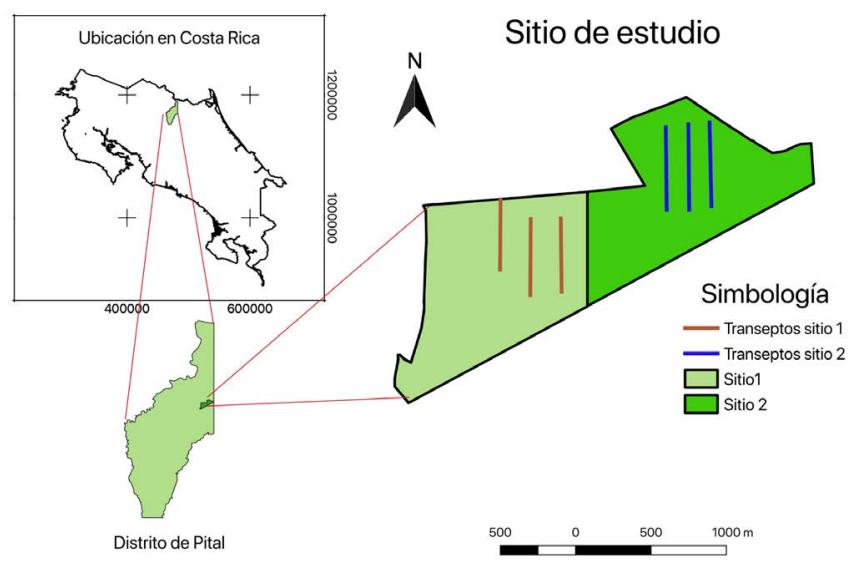

Figura 1. Ubicación de los sitios de estudio y distribución de los transeptos de muestreo.

Figure 1. Location of study sites and distribution of sampling transepts. 


\section{Transeptos de muestreo de mariposas}

Los transeptos de muestreo de mariposas fueron instalados en las áreas de bosque aprovechado, en cada sitio y la elección del punto de arranque para inicio de los transeptos, fue al azar.

Tanto en el Sitio 1 como en el Sitio 2, se establecieron 3 transeptos. El área de muestreo se distanció $150 \mathrm{~m}$ de los bordes del bosque que se encuentran adyacentes a áreas de potrero y la distancia entre transeptos también fue de $150 \mathrm{~m}$ para lograr independencia entre ellos. Los transeptos se distribuyeron a lo largo de cada una de las áreas de estudio, específicamente en las áreas de bosque donde se dio la mayor actividad de aprovechamiento. El total de distancia muestreada para las mariposas fue de $550 \mathrm{~m}$ por transepto y en los diferentes transeptos se atravesó por áreas de claros producidas por la caída de los árboles extraídos y no extraídos, áreas no alteradas por el aprovechamiento, áreas afectadas por caminos primarios y secundarios de aprovechamiento y áreas de protección hídrica.

El monitoreo de mariposas en el Sitio 1 y 2 se realizó 7 y 1,5 meses, respectivamente; después de concluida la intervención en cada sitio. El monitoreo de mariposas se realizó en el Sitio 1 y el Sitio 2 con una diferencia de 24 horas entre que se terminó de muestrear el Sitio 1 y se inició el monitoreo del Sitio 2.

\section{Monitoreo de mariposas}

El monitoreo de mariposas se realizó aproximadamente entre las 8:30 y las 14:30 horas, durante 5 días en cada sitio de estudio, para un total de 6 horas por día de muestreo, 2 horas por transepto, en condiciones de buen tiempo, sin lluvia y en la medida de lo posible con alta radiación, siguiendo la metodología de monitoreo ecológico del manejo forestal en el trópico húmedo [7]. Cada transepto fue recorrido caminando a una velocidad constante. Se registraron y contaron todos los individuos que pasaron frente al evaluador a un máximo de $5 \mathrm{~m}$ a cada lado de cada transepto, no se hizo visualizaciones hacia atrás, el esfuerzo de muestreo acumulado equivalente a 30 horas por sitio.

\section{Colecta, preservación e identificación de especímenes}

Fueron colectados todos los individuos desconocidos pertenecientes a las familias Nymphalidae, Papilionidae y Pieridae, para ello se utilizó una red de golpe. Luego, cada mariposa fue colocada en un sobre de papel pergamino, para la conservación de la mariposa, dispuesto en una caja plástica fría. Al colectar se anotó características relevantes del espécimen como color, tamaño, y otras características distintivas, además en el sobre se escribió un código, todo esto con el fin de utilizarlo en el proceso de identificación. La selección de las 3 familias de mariposas, elegidas para este estudio, se hizo siguiendo la recomendación brindada en la Guía de monitoreo de bosques de alto valor, la cual recomienda excluir las familias de difícil identificación [7].

La determinación taxonómica de los especímenes fue realizada en la Escuela de Biología de la Universidad de Costa Rica. Adicionalmente, se fotografió cada una de las mariposas y se conformó un archivo digital, con cada una de ellas identificadas. Las muestras de mariposas fueron depositadas en el Museo de Mariposas de la Universidad de Costa Rica, Campus Rodrigo Facio y en el Laboratorio de Entomología de la Escuela de Ingeniería Forestal del Tecnológico de Costa Rica, Campus Cartago.

\section{Análisis de datos}

Con los datos de las parcelas temporales de muestreo de cada sitio se calculó el área basal en metros cuadrados

Cuadro 1. Caracterización de los dos sitios de estudio ubicados en Yucatán de Pital de San Carlos, Alajuela, Costa Rica.

Table 1. Characterization of the two study sites located in Yucatán of Pital de San Carlos, Alajuela, Costa Rica.

\begin{tabular}{|c|c|c|c|c|}
\hline Sitio & $1^{*}$ & $2^{*}$ & $F$ & F crítica \\
\hline Área efectiva de manejo (ha) & 81,00 & $61,00^{\star \star \star}$ & - & - \\
\hline Área basal mayor a $10 \mathrm{~cm}\left(\mathrm{~m}^{2} \mathrm{ha}^{-1}\right)$ & $19,90 A$ & $23,90 A$ & 2,822 & 5,318 \\
\hline Cobertura de copa (\%) & $79,84 \mathrm{~A}$ & $71,90 B$ & 4,840 & 3,963 \\
\hline Humedad Relativa (\%) & $99,03 \mathrm{~A}$ & $98,99 A$ & 0,035 & 3,963 \\
\hline Temperatura $\left({ }^{\circ} \mathrm{C}\right)$ & $28,68 \mathrm{~A}$ & $27,82 \mathrm{~A}$ & 4,404 & 4,965 \\
\hline Tamaño de claros $\left(m^{2}\right)^{\star \star}$ & 175,3 & $>500$ & - & - \\
\hline
\end{tabular}

*Las letras diferentes entre sitios representan diferencias significativas al 95\% de confianza.

**Información obtenida de los informe de regencia emitidos para los sitios evaluados.

${ }^{* * \star}$ El plan de manejo aprobado se hizo por 61 ha pero al final el aprovechamiento se concentró en 30 ha que fue de donde se extrajo los árboles caídos por el huracán Otto. 

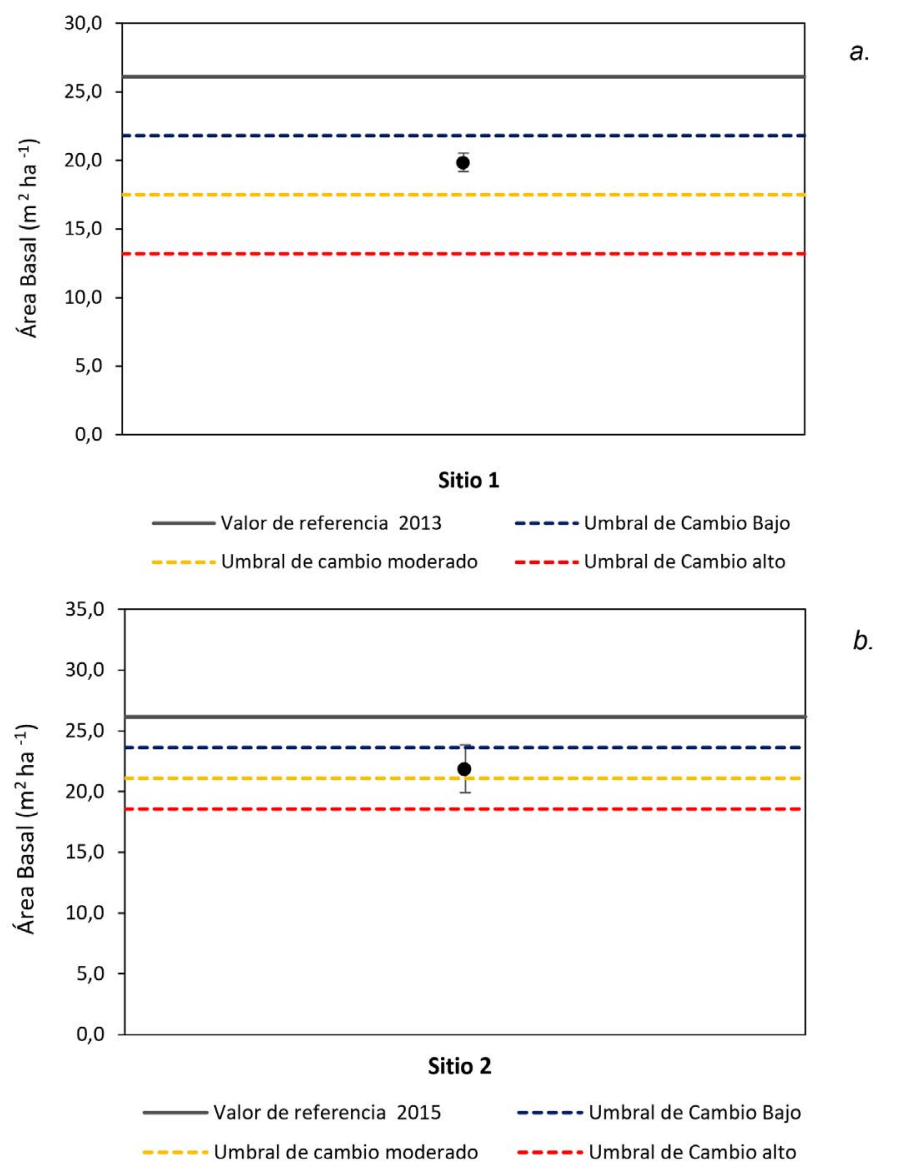

Figura 2. Umbrales de cambio en área basal arriba de $10 \mathrm{~cm}$ de diámetro para los sitios evaluados en Yucatán Pital de San Carlos, Alajuela, Costa Rica.

Figure 2. Thresholds of change in basal area above $10 \mathrm{~cm}$ in diameter for the sites evaluated in Yucatan Pital de San Carlos, Alajuela, Costa Rica.

por hectárea. Se utilizó el programa SPSS versión 23.0 [16], para generar la estadística descriptiva y el análisis de varianza de los sitios para el área basal, la temperatura, la humedad relativa y la cobertura forestal, todos al $95 \%$ de confianza. En el análisis de la cobertura forestal se utilizó el programa Gap Light Analizer 2.0 [17].

Para tomar la decisión sobre la determinación de línea base se analizó información de parcelas permanentes de muestreo establecidas en ambos sitios entre los años 1994 y 1995 [18] cuando los sitios no habían sido intervenidos e información de los planes de manejo diseñados para el segundo ciclo de corta. Para calcular los umbrales de cambio se utilizó la referencia de Finegan et al. [7] donde una vez definida la línea de referencia se considera que menos una desviación estándar corresponde con el cambio bajo, menos dos desviaciones estándar corresponden con cambio moderado y menos tres desviaciones estándar corresponden con cambio drástico.
La diversidad de las especies fue analizada obteniendo los índices de diversidad de Shannon $\left(H^{\prime}\right)$ y Simpson (1-D) y el índice de equitatividad $\mathrm{J}$ utilizando el programa Past versión 3.2. La diferencia entre sitios para los índices H'y 1-D fue realizada utilizando el mismo programa mediante pruebas $\mathrm{t}(a=0,05)$.

Las curvas de acumulación de especies fueron hechas con información de cada sitio en el programa EstimateS versión 9.1 utilizando los valores promedio (S) y CHAO1. Además, a partir de las curvas $\mathrm{CHAO1}$ se generaron las ecuaciones de mejor ajuste por métodos no paramétricos, obteniendo su primera derivada para calcular los puntos de inflexión y el total de especies en el punto de inflexión, reportando para cada una el $r^{2}$.

Se elaboró un listado de las preferencias de hábito de las diferentes especies de mariposa muestreadas utilizando la clasificación de Ordoñez [19], utilizando los reportes de DeVries [20], Young [21], Krenn et al. [22], Tobar [14], Vega [23] y Murillo-Hiller [24].

\section{Resultados}

\section{Sitios Evaluados}

Según la información recopilada y el análisis de varianza realizado $(a=0,05)$ al comparar los sitios, (Cuadro 1) no se encontró diferencias significativas en el área basal, en el porcentaje de humedad relativa y en la temperatura ambiental.

La única diferencia significativa encontrada entre los sitios fue en el porcentaje de cobertura forestal, en este caso, el Sitio 1 registra un porcentaje de cobertura forestal mayor que el Sitio 2. Los claros del Sitio 2 son más grandes que los del Sitio 1; además, los claros del Sitio 1 están distribuidos en 81 ha mientras que los del Sitio 2 están concentrados en un área de 30 ha.

Tanto en el Sitio 1 como en el Sitio 2 las especies arbóreas más abundantes son Pentaclethra macroloba y Dialium guianensis, la tercera más abundante en el Sitio 1 es el Carapa guianensis y en el Sitio 2 es Vochysia allenii.

\section{Umbrales de cambio en área basal}

Para efectos de esta evaluación se utilizó un indicador de filtro grueso como lo es el área basal [7]. En el Sitio 1 la primer línea de referencia confirmada es la que corresponde con el bosque en completo estado natural antes de cualquier intervención (1994), el área basal registrada en ese momento, para árboles arriba de $10 \mathrm{~cm}$ de diámetro, fue de $30,33 \mathrm{~m}^{2} \mathrm{ha}^{-1}$ ( $\mathrm{s}=3,35$ y $\mathrm{CV}=11,1 \%$ ). En el año 2013 registró un área basal de $26,12 \mathrm{~m}^{2} \mathrm{ha}^{-1}$ ( $s=4,31$ y $C V=16,6 \%)$. El área basal post intervención 
Cuadro 2. Distribución de la riqueza y abundancia de especies de mariposas de las familias y subfamilias de interés durante el período de muestreo en los sitios de interés Yucatán Pital de San Carlos, Costa Rica.

Table 2. Distribution of the richness and abundance of species of butterflies of the families and subfamilies of interest during the sampling period in the sites of interest Pital of San Carlos, Costa Rica.

\begin{tabular}{|c|c|c|c|c|c|c|c|c|c|}
\hline \multirow{2}{*}{ Familia } & \multirow{2}{*}{ Subfamilia } & \multicolumn{2}{|c|}{ Sitio 1} & \multicolumn{2}{|c|}{ Riqueza } & \multicolumn{2}{|c|}{ Sitio 2} & \multicolumn{2}{|c|}{ Riqueza } \\
\hline & & Abundancia & $\%$ & No spp & $\%$ & Abundancia & $\%$ & No spp & $\%$ \\
\hline \multirow{7}{*}{ Nymphalidae } & Biblidinae & 0 & 0,0 & 0 & 0,0 & 1 & 0,6 & 1 & 4,0 \\
\hline & Charaxinae & 1 & 1,1 & 1 & 6,3 & 3 & 1,7 & 1 & 4,0 \\
\hline & Cyrestinae & 0 & 0,0 & 0 & 0,0 & 3 & 1,7 & 1 & 4,0 \\
\hline & Danainae & 1 & 1,1 & 1 & 6,3 & 17 & 9,4 & 4 & 16,0 \\
\hline & Heliconiinae & 37 & 41,6 & 5 & 31,3 & 64 & 35,4 & 9 & 36,0 \\
\hline & Nymphalidae & 1 & 1,1 & 1 & 6,3 & 4 & 2,2 & 1 & 4,0 \\
\hline & Satyrinae & 42 & 47,2 & 5 & 31,3 & 39 & 21,5 & 5 & 20,0 \\
\hline Papilionidae & Papilioninae & 6 & 6,7 & 2 & 12,5 & 33 & 18,2 & 2 & 8,0 \\
\hline Pieridae & Pierinae & 1 & 1,1 & 1 & 6,3 & 17 & 9,4 & 1 & 4,0 \\
\hline Total & & 89 & 100,0 & 16 & 100,0 & 181 & 100,0 & 25 & 100,0 \\
\hline
\end{tabular}

fue de $19,8 \mathrm{~m}^{2} \mathrm{ha}^{-1}(\mathrm{~s}=0,7$ y $\mathrm{CV}=3,5 \%)$. Para efectos de construir los umbrales de cambio bajo, moderado y alto (figura 2a.) se tomó la decisión de utilizar de referencia el área basal obtenida en el año 2013, antes de que ocurriera el segundo aprovechamiento del que se tiene conocimiento en el Sitio 1, esto por cuanto no se tiene información de parcelas permanentes que hayan monitoreado el área basal en zonas no perturbadas que permitan medir el cambio natural del Sitio 1 entre 1994 y el 2013. Puede observarse en la Figura 2a. que el área basal registrada un año después del cierre del aprovechamiento (2017) ubica el bosque del Sitio 1 entre los umbrales de cambio moderado y cambio bajo, cuyas barras de error no tocan ninguna de los dos activadores. Es importante hacer notar que esa área basal refleja el efecto del aprovechamiento más el efecto de los daños producidos por el Huracán Otto.

En el caso del Sitio 2, igualmente la primer línea de referencia confirmada es la que corresponde con el bosque en completo estado natural antes de cualquier intervención (1995), el área basal registrada en ese momento, para árboles arriba de $10 \mathrm{~cm}$ de diámetro, fue de $27,9 \mathrm{~m}^{2} \mathrm{ha}^{-1}$ (s=3,14 y CV=11,3\%). En el año 2015 el área basal antes del aprovechamiento fue de $26,14 \mathrm{~m}^{2}$ ha $^{-1}(s=2,52$ y CV=10,4\%). Después del aprovechamiento el área basal registrada es de $21,9 \mathrm{~m}^{2} \mathrm{ha}^{-1}(\mathrm{~s}=2,0 \mathrm{y}$ $\mathrm{CV}=9,13 \%$ ). Para efectos de construir los umbrales de cambio bajo, moderado y alto (figura 2b.) del Sitio 2, también se tomó la decisión de utilizar de referencia el área basal obtenida en el año 2015, antes de que ocurriera el segundo aprovechamiento del que se tiene conocimiento, esto por cuanto no se tiene información de parcelas permanentes que hayan monitoreado el área basal en zonas no perturbadas que permitan medir el cambio natural del Sitio 2.
Puede observarse en la figura $2 \mathrm{~b}$ que el área basal registrada, 1,5 meses después del aprovechamiento, ubica el bosque del Sitio 2 entre los umbrales de cambio moderado y cambio bajo; sin embargo, en este caso las barras de error traspasan hacia arriba los umbras del cambio bajo a valor de referencia y en la parte baja los traspasa del umbral de cambio moderado al cambio alto. Es importante hacer notar también que esa área basal refleja el efecto del aprovechamiento más el efecto de los daños producidos por el Huracán, el cual no fue posible evaluar por separado.

\section{Composición y riqueza de mariposas}

La familia Nymphalidae, cuadro 2 , fue la familia que más individuos registró, en el Sitio 1 representa el 92\% de la abundancia total, mientras que en el Sitio 2 representa el $88 \%$. Dentro de esta familia, en el Sitio 1 se logra ubicar 13 especies, mientras que el Sitio 2 concentró 22 especies diferentes. También puede observarse que dentro de la Nymphalidae las dos subfamilias más representadas son Satyrinae con las especies Antirrhea philoctetes lindigii, Cissia confusa, C. hesione, C. usitata, Dulcedo polita, Euptychia westwoodi, Morpho melenaus, Pierella helvina incanescens, y Heliconiinae específicamente con las especies Heliconius cygno galanthus, $H$. erato, $H$. sapho leuce y H.sara fulgidus, entre otras. Ambas subfamilias representan en el Sitio 1 un $88,8 \%$ de la abundancia y un $62,6 \%$ de la riqueza. En el Sitio 2, estas mismas dos subfamilias representan el $56,9 \%$ de la abundancia y el $56,0 \%$ de la riqueza. En el Sitio 2 también resulta bastante abundante la familia Papilionidae con un $18,2 \%$ de la abundancia, concentrada en dos especies que representan solo el $8 \%$ de la riqueza y que está representada por Parides childrenae y $P$. eurimedes mylotes, ambas especies 
Cuadro 3. Abundancia de especies y reporte de hábito por especies según sitio para las familias de interés muestreadas en dos sitios ubicados en Yucatán de Pital de San Carlos Alajuela, Costa Rica.

Table 3. Abundance of species and habit report by species according to site, for families of interest sampled at two sites located in Pital Yucatan, Alajuela, Costa Rica.

\begin{tabular}{|c|c|c|c|}
\hline Especie & Sitio 1 & Sitio 2 & Hábitat \\
\hline Aeria eurimedia agna & 0 & 5 & Sotobosque sombreado \\
\hline Antirrhea philoctetes lindigii & 0 & 1 & Sotobosque sombreado \\
\hline Cissia confusa & 1 & 0 & Sotobosque sombreado \\
\hline Cissia hesione & 0 & 2 & Sotobosque abierto \\
\hline Cissia usitata & 0 & 25 & Sotobosque abierto \\
\hline Dulcedo polita & 5 & 0 & Sotobosque sombreado \\
\hline Eresia mechanitis & 1 & 4 & Generalista \\
\hline Eueides aliphera & 0 & 4 & Bordes, claros grandes y áreas disturbadas \\
\hline Eueides vibilia vialis & 4 & 15 & Sotobosque sombreado \\
\hline Euptychia westwoodi & 1 & 0 & Sotobosque abierto \\
\hline Hamadryas arinome ariensis & 0 & 1 & Generalista \\
\hline Heliconius cydno galanthus & 5 & 2 & Generalista \\
\hline Heliconius erato & 0 & 2 & Bordes, claros grandes y áreas disturbadas \\
\hline Heliconius sapho leuce & 17 & 20 & Sotobosque sombreado \\
\hline Heliconius sara fulgidus & 10 & 13 & Bordes, claros grandes y áreas disturbadas \\
\hline Hypna clytemnestra & 0 & 2 & Sotobosque sombreado \\
\hline Hypoleria cassotis & 1 & 0 & Sotobosque sombreado \\
\hline Laparus doris viridis & 0 & 1 & Sotobosque abierto \\
\hline Lycorea halia atergatis & 0 & 11 & Bordes, claros grandes y áreas disturbadas \\
\hline Marpesia merops & 0 & 3 & Dosel y claros \\
\hline Mechanitis polymnia isthmia & 1 & 5 & Generalista \\
\hline Melinaea ethra lilis & 0 & 1 & Generalista \\
\hline Morpho melenaus & 21 & 5 & Todos menos generalista \\
\hline Philaethria dido & 0 & 2 & Dosel y claros \\
\hline Pierella helvina incanescens & 14 & 6 & Sotobosque sombreado \\
\hline Zaretis itys & 1 & 1 & Generalista \\
\hline Parides childrenae & 1 & 8 & Sotobosque abierto \\
\hline Parides eurimedes mylotes & 5 & 25 & Sotobosque abierto \\
\hline Appias drusilla & 1 & 17 & Bordes, claros grandes y áreas disturbadas \\
\hline Total & 89 & 181 & \\
\hline
\end{tabular}

Cuadro 4. Puntos de inflexión y número de especies calculados a partir de las funciones de mejor ajuste para los modelos no paramétricos generados para las curvas reales y CHAO en los sitios evaluados en Yucatán Pital de San Carlos, Costa Rica.

Table 4. Inflection points and number of species calculated from the best fit functions for the non-parametric models generated for the real and $\mathrm{CHAO}$ curves at the evaluated sites in Pita de San Carlos, Costa Rica.

\begin{tabular}{cccccc|} 
Curva & Función de mejor ajuste & Primera derivada & $\begin{array}{c}\text { Inflexión } \\
\text { (horas) }\end{array}$ & $\begin{array}{c}\text { Número de } \\
\text { especies }\end{array}$ & $r^{2}$ \\
\hline Real & & & & & \\
\hline Sitio 1 & $F(x)=-0,0058 x^{2}+0,5655 x+4,12$ & $F^{\prime}(x)=-0,0116 x+0,5655$ & 49 & 18 & 0,9978 \\
\hline Sitio 2 & $F(x)=-0,0183 x^{2}+1,2871 x+5,68$ & $F^{\prime}(x)=-0,077 x+2,0555$ & 35 & 28 & 0,9987 \\
CHAO 1 & & & & & \\
\hline Sitio 1 & $F(x)=0,034 x^{2}-0,0204 x+13,936$ & $F^{\prime}(x)=-0,068 x-0,0204$ & 0,3 & 16 & 0,9966 \\
\hline Sitio 2 & $F(x)=-0,0343 x^{2}+1,7732 x+7,456$ & $F^{\prime}(x)=-0,0686 x+1,7732$ & 26 & 30 & 0,9876 \\
\hline
\end{tabular}


Cuadro 5. Valores medios de los Índices de diversidad calculados para la comunidad de mariposas evaluadas en dos sitios.

Table 5. Mean values of the diversity indices calculated for the community of butterflies evaluated in two sites.

\begin{tabular}{llll}
\hline Índices & Sitio 1 & Sitio 2 & $\mathrm{P}$ \\
\hline Shannon H & 2,222A & 2,755B & 0,0001 \\
Simpson 1-D & 0,858A & 0,918B & 0,002 \\
\hline
\end{tabular}

*Las letras diferentes entre sitios representan diferencias

significativas al 95\% de confianza.

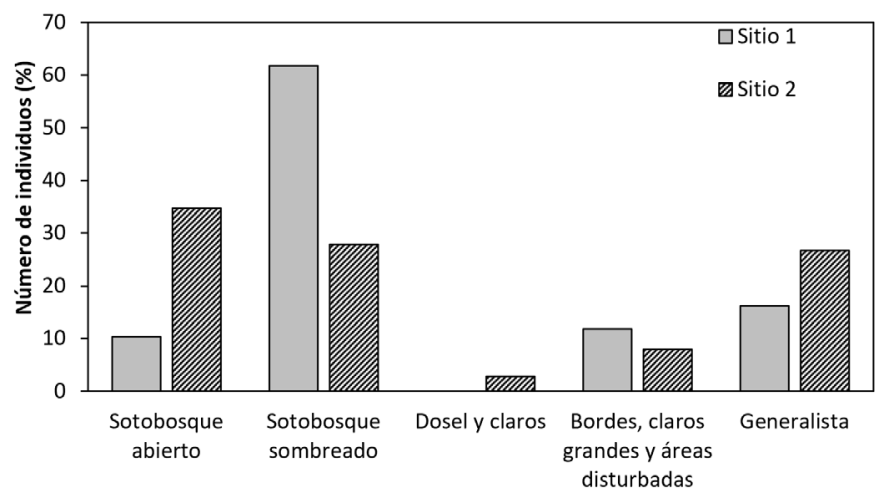

Figura 3. Número total de mariposas registradas (\%) según su hábitat característico para los dos bosques evaluados.

Figure 3. Total number of registered butterflies (\%) according to their characteristic habitat for the two forests evaluated.

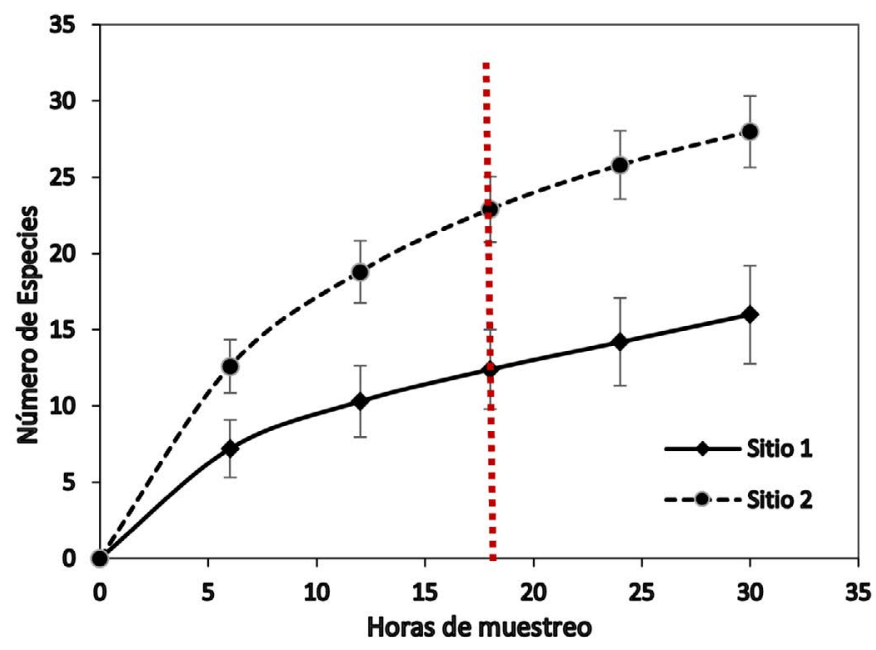

Figura 4. Curvas de acumulación de especies de mariposas observadas en transeptos de $550 \mathrm{~m}$ para los dos sitios de estudio (100 aleatorizaciones).

Figure 4. Curves of accumulation of species of butterflies observed in transects of $550 \mathrm{~m}$ for the two study sites (100 randomizations). características de los sotobosques abiertos.

En el Sitio 1 se colectaron un total de 81 mariposas, de las 3 familias estudiadas, distribuidas en 16 especies, siendo la más abundante la especie Morpho melenaus, seguida por Heliconius sapho leuce y Pierella helvina incanescens. En el Sitio 2 se colectaron 181 individuos distribuidos en 25 especies diferentes. Las especies más abundantes en este Sitio 2 fueron Parides eurimedes mylotes, Cissia usitata y Heliconius sapho leuce. (cuadro 3).

Como puede observarse en la figura 3 , de las especies observadas en el Sitio 1, un $62,1 \%$ de los individuos corresponden con especies presentes en el sotobosque sombreado, con las especies Cissia confusa, Dulcedo polita, Eueides vibilia vialis, Heliconius sapho leuce, Hypoleria cassotis y Pierella helvina incanescens, mientras que en el Sitio 2 esa misma proporción se alcanza sumando las especies de sotobosque abierto con Cissia hesione, C. usitata, Laparus doris viridis, Parides childrenae y $P$. eurimedes mylotes más especies de sotobosque sombreado como Aeria eurimedia agna, Antirrhea philoctetes lindigii, Eueides vibilia vialis, Heliconius sapho leuce, Hypna clytemnestra y Pierella helvina incanescens. También es observable que los individuos de especies generalistas tales como Eresia mechanitis, Hamadryas arinome ariensis, Mechanitis polymnia isthmia y Melinaea ethra lilis se presentan en mayor proporción y algunas solo en el Sitio 2 (cuadro 3). En ambos sitios se puede observar que las especies que se clasifican como de dosel y claros ocupan una menor proporción en el Sitio 2 y en el Sitio 1 no se identificaron.

\section{Curvas de acumulación}

En la figura 4 se muestran las dos curvas de acumulación de especies de ambos sitios, las cuales permiten no solo observar la riqueza de los sitios sino también inferir sobre el muestreo realizado y el tiempo necesario para conocer las especies del lugar Además, puede observarse que aunque ambos sitios recibieron manejo forestal, el sitio con mayor abundancia de mariposas es el que recién ha terminado la intervención, que corresponde con el Sitio 2.

Al generar las curvas se observa que posterior a las 8 horas de muestreo hay un cambio de pendiente en la curva mostrando de ahí en adelante una menor cantidad de especies nuevas. Tanto en el Sitio 1 como en el Sitio 2 , el $75 \%$ de las especies nuevas se muestrearon antes de las 18 horas de muestreo acumulado. En el Sitio 1 se alcanza un total de 16 especies a las 30 horas de muestreo mientras que en el mismo tiempo el Sitio 2 alcanza un total de 27 especies.

Según las curvas CHAO 1, para el Sitio 1 se esperarían 16 especies y para el Sitio 2 se esperarían 30 especies. En 
el cuadro 4 se presentan las fórmulas obtenidas a partir de las curvas de mejor ajuste generadas por métodos no paramétricos, y su primera derivada, así como los puntos de inflexión y el total de especies calculado en el punto de inflexión, todas las curvas con $\mathrm{r}^{2}$ muy cercano a 1.

\section{Índices de diversidad}

El índice de Shannon dio como resultado 2,22 para el Sitio 1 y 2,76 para el Sitio 2. El análisis de comparación entre ambos sitios demuestra que existen diferencias significativas entre los dos sitios. La misma tendencia se confirma con el índice de Simpson 1-D, (ver cuadro 5) donde se confirma que el sitio más diverso en mariposas es el Sitio 2. Por su parte, la Equitatividad $\mathrm{J}$ para el Sitio 1 es de 0,801 y para el Sitio 2 es de 0,856. Puede observarse que hay mayor equitatividad en el Sitio 2 que en el Sitio 1.

\section{Discusión}

\section{Sitios Evaluados}

El hecho de que no se encontraran diferencias significativas en la estructura del bosque y en los promedios de temperatura y humedad relativa a nivel del piso del bosque, demuestra que ambos sitios, desde un punto de vista estadístico, son similares; no obstante, sí se ha obtenido una diferencia significativa en el porcentaje de cobertura forestal entre ambos sitios. Esta diferencia significaría que a pesar de que ambos bosques son similares en los aspectos biofísicos, el efecto sumado del aprovechamiento y el efecto del paso del Huracán Otto ha dejado condiciones diferentes en cuanto al porcentaje de cobertura forestal, lo cual podría explicarse por dos razones, la primera de ellas es el tiempo de recuperación que ha transcurrido en cada sitio desde la última perturbación, en el Sitio 1, ese tiempo es mayor que en el Sitio 2. La segunda es la distribución y tamaño de los claros pues en áreas afectadas por huracanes los claros pueden llegar incluso a ser de más de $1600 \mathrm{~m}^{2}$ [25] aumentando el área expuesta a la radiación solar directa hasta los estratos inferiores del bosque, incluso el suelo, generando un cambio drástico especialmente en la luminosidad de estas áreas, lo que influye no solo en el tipo de vegetación que se establecerá sino en la fauna que frecuentará estas áreas, como es el caso de las mariposas.

En el Sitio 2, es común observar árboles descopados, éstos se encuentran en pie aún vivos, pero con una ausencia de total a parcial de su copa lo que genera una exposición lumínica alta para los estratos inferiores, este daño es muy común cuando hay afectación por huracanes tal como lo reporta Vandermeer et al. [24] y Louman et al. [25].
Con respecto a la segunda razón, en el Sitio 1 algunos caminos primarios y secundarios están obstruidos con los árboles caídos y los claros producidos por árboles caídos por el huracán están en proceso de cicatrización al igual que los claros producidos por el aprovechamiento, pero en el Sitio 2, la caída de árboles por efecto del huracán se concentró en ciertas áreas, esto hace que los claros no solo estén más cercanos entre sí; sino que sean más grandes de lo que se produce en un aprovechamiento normal, esta afectación es parte de los riesgos que se corren por las condiciones del medio en general [1] y es un riesgo común además cuando hay afectación por huracanes [25].

\section{Umbrales de cambio del área basal}

En el caso del Sitio 1 y el Sitio 2 las medias de área basal se ubican en los umbrales de cambio bajo a moderado, parte de lo que explica la posición de estos valores en ese rango tiene que ver con los efectos sumados del aprovechamiento y de la caída de árboles por efectos del paso del huracán Otto, en el año inmediatamente posterior a la intervención se dan las mayores pérdidas de área basal pues muchos árboles que no han sido aprovechados, especialmente de bajas dimensiones mueren por los efectos secundarios de la intervención, árboles que quedan quebrados, descopados parcial o totalmente o que simplemente mueren por el exceso de luminosidad producida por los claros y particularmente en especies que no son tolerantes [27], [28]. Es evidente que el efecto de la distribución y frecuencia de los claros produce un efecto importante en el área basal pues la desviación estándar del área basal en el Sitio 1 es muy baja pero en el Sitio 2 es mayor, lo que incluso hace que el área basal supere los activadores de umbral de cambio moderado a cambio alto o bien los activadores de cambio entre la línea de referencia y el cambio bajo. Esto demuestra que en el Sitio 2 podrían localizarse zonas con diferente grado de perturbación, aspecto que ya se mencionó cuando se discutió las diferencias de porcentaje de cobertura de ambos sitios.

Más importante aún es indicar que con los resultados obtenidos queda demostrado que es necesario ajustes al manejo particularmente en la definición del nuevo ciclo de corta, de tal forma que permitan la recuperación de las áreas pues el impacto sumado del aprovechamiento y del paso del huracán Otto, es mayor de lo aceptable [7], [19], [11], y en consecuencia será necesario más tiempo para recuperar las condiciones del sitio lo más cercano a lo encontrado antes de las intervenciones. A pesar de esto, no puede atribuirse todo el impacto al aprovechamiento pues en un bosque ubicados a 3 $\mathrm{km}$ de distancia de los dos sitios evaluados que no fue aprovechado, las pérdidas de área basal ascendieron a un 30\% del área basal original para individuos mayores a $10 \mathrm{~cm}$ de diámetro solo por efecto del huracán Otto, [29], es evidente que los efectos de un fenómeno natural 
como lo es un huracán genera en el Sitio 2 , más que en el Sitio 1, una desigualdad en la distribución del área basal que solo el tiempo logrará cicatrizar.

\section{Composición y riqueza de mariposas}

El monitoreo de mariposas es considerado un muestreo de filtro fino [7]. En este caso, aunque no fue posible localizar un sitio no perturbado cercano que permitiera definir la línea de referencia sobre las mariposas presentes para los dos sitios muestreados que permitiera definir el nivel de referencia para la composición y riqueza de las 3 familias elegidas en este monitoreo, sí es posible utilizar los resultados de este monitoreo para comparar con monitoreos sucesivos y con ellos determinar la variación y el comportamiento de estos parámetros en el tiempo.

El Sitio 2 es el que registra una mayor abundancia de mariposas y también mayor cantidad de especies distribuidas en las 3 familias analizadas, es de notar que las tres especies más abundantes de este sitio representan el $39 \%$ de la abundancia total de este sitio y que dos de estas especies corresponde con especies de sotobosques abiertos. Pero en el Sitio 1 esta situación es diferente, primero porque las 3 especies más abundantes representan el $58,4 \%$ de la abundancia total y segundo porque las 3 especies corresponden con mariposas características de sotobosque cerrado lo que reafirma que el bosque del Sitio 1 tienen una menor perturbación que el bosque del Sitio 2. Comparando los resultados de este muestreo con los obtenidos en bosques muy húmedos tropicales bajo manejo forestal en Nicaragua y reportados por Ordoñez [19] y Ordoñez et al. [11], los resultados son bastante diferentes, primero porque la composición en general reportada para sitios 1 y 2 es diferente a lo que se ha reportado en Nicaragua y segundo porque las mariposas más abundantes en los sitios estudiados en Costa Rica corresponden con mariposas de sotobosque sombreado. Además, aunque ambos estudios tienen esfuerzos de muestreo de 30 horas, [19], [11] realizaron su muestreo en un período que comprendió 3 meses, de marzo a junio, mientras que el presente estudio realizó su trabajo de campo durante 3 semanas en los meses de junio-julio; la composición vegetal del sitio, el tipo de planta hospedera y las épocas de floración son parte de las razones por las cuales la presencia y composición de mariposas puede ser diferente [30], [27], [40], [41].

\section{Curvas de acumulación}

Las curvas de acumulación no solo permiten conocer la riqueza de los sitios sino inferir sobre el muestreo realizado [39]. Los resultados muestran que a las 18 horas de muestreo ya se ha muestreado el $75 \%$ de los especímenes que se esperan y a las 30 horas son pocos los cambios en el esfuerzo de muestreo que se esperarían, y por ello se sugiere que el muestreo realizado ha sido suficiente, estos resultados son similares a los obtenidos por [19] para bosques muestreados en Nicaragua, los cuales reflejaron pocos cambios arriba de las 20 horas de muestreo. No existen muestreos similares en bosque, publicados para la zona de estudio en Costa Rica. El mayor número promedio de especies en el Sitio 2 se confirma no solo con los resultados de riqueza del mismo sitio, sino que también coinciden con el bosque en el que al momento del muestreo tienen una mayor perturbación reflejada en una mayor exposición a la radiación solar por el tamaño de los claros y la frecuencia de los mismos, producto del efecto combinado entre la perturbación producida por el manejo forestal realizado y por los efectos del huracán Otto, esta tendencia es similar a lo determinado también por [19] en un bosque en Nicaragua que tuvo un manejo forestal de alto impacto.

\section{Índices de diversidad}

Según el índice de Shannon el Sitio 2 registra una diversidad significativamente mayor que la del Sitio 1. Esto puede deberse a que el Sitio 2 también tiene un porcentaje de cobertura significativamente menor que el Sitio 1 y tal como lo han confirmado los estudios de [19], [12]-[14], [31]-[34], en los bosques con menor cobertura y mayor cantidad de claros se desarrollan una serie de variaciones microclimáticas tanto horizontal como verticalmente en el bosque, lo que permite que mariposas de especies de sitios abiertos y generalistas aumenten dentro del bosque, aumentando significativamente la diversidad. Esto significa que dependiendo de cómo es la estructura vegetal, así es la composición y abundancia de las especies de mariposas presentes [35]. La misma tendencia que muestra el índice de Shannon es confirmada por el índice de Simpson. Demostrándose que en los bosques con mayor perturbación se alcanzan mayor diversidad [35]. Con el índice de equitatividad J se demuestra que el Sitio 2 tienen una mayor equitatividad; no obstante tanto para el Sitio 2 como para el Sitio 1 ese valor es mayor a 0,8 y esto significa que hay una muy alta equitatividad en la representación de los individuos de cada especie detectada en el muestreo, [36] demostrando que para ambos sitios la diversidad muestreada es muy cercana a la diversidad máxima esperada [42]. Utilizando todos los índices calculados es demostrable que el Sitio 2 es el más diverso y el de mayor equitatividad, lo que es una respuesta lógica dado que como consecuencia de la perturbación se generan una serie de micro hábitats que logran atraer mariposas del borde del bosque, del dosel y de áreas abiertas [37], [38], [14]. Como ya se ha dicho, la frecuencia y el tamaño de los claros en el Sitio 2 es mayor y eso permite la llegada de especies generalistas y de sotobosque abierto en mayor cantidad. 


\section{Conclusiones y recomendaciones}

El área basal del bosque como indicador de sostenibilidad en la categoría de monitoreo ecológico de filtro grueso fue posible y sencillo de utilizar. Es importante utilizar información que tengan errores de muestreo inferiores al $20 \%$ con el fin de asegurar calidad de la información analizada.

El monitoreo con indicadores de filtro fino como lo son las mariposas, es sencillo de aplicar en el campo pero las líneas dereferencianoson fáciles de construir debidoaque la composición, abundancia y riqueza de las mariposas varía a lo largo del año y además es dependiente de la vegetación existente y de si los bosques son unidades de manejo aisladas o por el contrario forman parte de un conjunto de bosque mayor. Desde este punto de vista para lograr utilizar con eficiencia el monitoreo ecológico de mariposas como predictor el MFS, es necesario un esfuerzo mayor y coordinado entre los propietarios del bosque, los tomadores de decisión sobre el manejo y las autoridades estatales, que permita construir línea de referencia confiables para este tipo de indicador.

Se encontró diferencias significativas en la abundancia y composición de las mariposas de los sitios evaluados pero de manera inversa se encontró diferencias significativas en el porcentaje de cobertura forestal de ambos sitios. Es decir a menor cobertura forestal, mayor abundancia y riqueza de mariposas.

Se encontró que en los sitios que tienen menos tiempo desde la última perturbación es más probable encontrar mariposas propias de sotobosques abiertos y especies generalistas. La tendencia sugiere que a un año de la última perturbación el bosque podría ser capaz de tener una riqueza y una abundancia similar a los bosques no perturbados donde dominan más las especies propias del sotobosque cerrado o del bosque continuo, pero este aspecto debe ser confirmado con resultados de monitoreo futuros.

No existen líneas de referencia para la abundancia y composición de mariposas, construidas a partir de bosques de referencia no perturbados, no fue posible establecer umbrales para estos parámetros; sin embargo, este muestreo podría servir de referencia para futuros monitoreos en los Sitios 1 y 2 que permitan no solo mostrar la evolución en la riqueza y abundancia de las mariposas sino monitorear la relación de la abundancia, la riqueza y la composición con el estado de recuperación de la cobertura forestal del bosque conforme van cicatrizando los claros.

En los sitios muestreados no fue posible separar los efectos del huracán de los efectos del aprovechamiento, por ello es importante realizar monitoreos futuros en la vegetación arbórea con el fin de determinar si el área basal del bosque del Sitio 2 se recupera de una manera adecuada, con el fin de asegurar que es posible un MFS en ciclos de corta de 15 años y si la recuperación no se lograra será necesario ajustar el ciclo de corta a un tiempo mayor, para asegurar una recuperación para este bosque.

Se recomienda que para estudios sucesivos evaluar la periodicidad de los muestreos ecológicos y el impacto económico que pudiera tener la realización de este tipo de monitoreo en la rentabilidad del MFS.

\section{Agradecimientos}

Las autoras agradecen a la Vicerrectoría de Investigación y Extensión del TEC, por el financiamiento del proyecto "Diseño de una metodología para Evaluar el Manejo Sostenible de los Bosques Naturales de la Región de La Cureña, Costa Rica" dentro del cual se desarrolló esta investigación, además se agradece a la oficina de Becas por el apoyo para financiar parte del trabajo de campo. Se agradece la colaboración al Lic Mario Quesada Quirós, la M.Sc. Casia Soto Montoya y el Dr. Ruperto Quesada Monge del TEC, por el apoyo en el trabajo de campo y al M.Sc. Ricardo Murillo-Hiller por el apoyo en la identificación de los especímenes de mariposa colectados y a Carlos Loría Murillo, Carlos Fernández Redondo y Álvaro Víquez Vargas por facilitar el acceso a las fincas.

\section{Referencias}

[1] O. A. Aguirre-Calderón, "Manejo forestal en el siglo XXI". Madera y bosques, 21, pp. 17-28. 2015.

[2] K. G. MacDicken, P. Sola, J. E. Hall, C. Sabogal, M. Tadoum, and C. Wasseige, C. "Global progress toward sustainable forest management." Forest Ecology and Management, 352, pp. 47-56. 2015.

[3] SINAC (Sistema Nacional de Áreas de Conservación), SIREFOR (Sistema de información de los recursos naturales), MINAE (Ministerio de Ambiente y Energía). Reporte estadístico forestal 2013, San José, Costa Rica: Cooperación Alemana Deutsche Zusammenarbetit, GIZ, 2013.

[4] Leyes y Decretos. "Ley Forestal No 7575", Asamblea Legislativa Costa Rica, 1996.

[5] SINAC (Sistema Nacional de Áreas de Conservación). "SINAC en números: informe anual de estadísticas SEMEC 2015", 2016. Disponible en: http://www.sinac.go.cr/ENUS/statistics/SINAC\%20in\%20Numbers/Informe\%20 SEMEC\%202015.pdf

[6] G. Galloway, S. Kengen, B. Louman, D. Stoian, G. Mery, F. Carrera-Gambeta y M. Trevin. "15 Cambios en los paradigmas del sector forestal de América Latina", Forest 
in the Global Balance-Changing Paradigms. IUFRO World Series. 17, 2016.

[7] B. Finegan, J. P. Hayes, D. Delgado y S. Gretzinger, "Monitoreo ecológico del manejo forestal en el trópico húmedo: una guía para operadores forestales y certificadores con énfasis en Bosques de Alto Valor para la Conservación", WWF centroamérica, 2004.

[8] S. E. Otavo, A. Parrado-Rosselli y J. A. Noriega," Superfamilia Scarabaeoidea (Insecta: Coleoptera) como elemento bioindicador de perturbación antropogénica en un parque nacional amazónico", Revista de Biología Tropical, vol. 61, no. 2, pp. 735-752, 2013.

[9] G. Andrade, "Utilización de las mariposas como bioindicadoras del tipo de hábitat y su biodiversidad en Colombia", Revista de la Academia Colombiana de Ciencias Exactas, Físicas y Naturales, vol. 22, no.84, pp. 407-421, 1998.

[10] J. Gerlach, M. Samways y J. Pryke, "Terrestrial invertebrates as bioindicators: an overview of available taxonomic groups", Journal of insect conservation, vol. 17, no. 4, pp. 831-850, 2013.

[11] Y. Ordoñez, D. Delgado y B. Finegan, "Monitoreo ecológico en bosques húmedos tropicales certificados en la RAAN, Nicaragua: evaluación del impacto ecológico del manejo forestal", Recursos Naturales y Ambiente, vol. 46, no. 4647, pp. 66-78, 2006.

[12] P. J. De Vries, D. Murray y R. Lande, "Species diversity in vertical, horizontal, and temporal dimensions of a fruitfeeding butterfly community in an Ecuadorian rainforest", Biological Journal of the Linnean Society, vol. 62, no. 3, pp. 343-364, 1997.

[13] N. Aguilar-Amuchástegui, "Criterios e indicadores de sostenibilidad ecológica: caracterización de la respuesta de dos grupos de insectos propuestos como verificadores", Tesis, Centro Agronómico Tropical de Investigación y Enseñanza, CATIE, Turrialba, Costa Rica, 1999.

[14] D. Tobar, "Efecto del Hábitat sobre la Comunidad de Mariposas Diurnas en un Paisaje Fragmentado del Norte de Costa Rica", Tesis. Centro Agronómico Tropical de Investigación y Enseñanza, CATIE, Turrialba, Costa Rica, 2004.

[15] A. Promis y G. Cruz. "Fotografías hemisféricas: un método para estimar estructuras del dosel arbóreo e iluminación en el interior del bosque". Revista Bosque Nativo, no. 44, pp 12-15, 2009.

[16] IBM. SPSS Statistic. SPSS 23.0 for windows, Chicago. USA. 2014.

[17] G. W. Frazer, C. D. Canham, K. P. Lertzman. Gap Light Analyzer (GLA) 2.0: Imaging software to extract canopy structure and gap light transmission indices from truecolour fisheye photographs. Burnaby, British Columbia: Simon Fraser University. 1999.

[18] E. Esquivel y C. Salas. "Índice de competencia individual en árboles del bosque húmedo tropical”. (Tesis de Bachillerato). Instituto Tecnológico de Costa Rica, Cartago, Costa Rica. pp. 45,1994.

[19] Y. Ordoñez, "Validación de indicadores ecológicos para la evaluación de Sostenibilidad en Bosques bajo manejo forestal en el trópico húmedo, con énfasis en Bosques de Alto Valor para la Conservación", tesis, Centro Agronómico Tropical de Investigación y Enseñanza, CATIE, Turrialba, Costa Rica, 2003.

[20] P. J. De Vries, "Butterflies of Costa Rica and their Natural History: Papilionidae, Pieridae, Nymphalidae". New Jersey: Princeton, 1987.

[21] A. M. Young, A. M. "The biology of the butterfly Aeria eurimedea agna (Nymphalidae: Ithomiinae: Oleriini) in Costa Rica". Journal of the Kansas Entomological Society, vol. 51, no. 1, pp. 1-10, 1978.

[22] H. W. Krenn, M. Wiemers, L. Maurer, V. Pemmer, W. Huber, A. Weissenhofer, "Butterflies of the Golfo Dulce region, Costa Rica", Verein zur Förderung der Tropenstation La Gamba, Vienna, 2010.

[23] G. Vega-Araya, "Guía de plantas hospederas para mariposarios", Santo Domingo, Heredia, Costa Rica: INBIO, p. 184, 2010.

[24] R. Murillo-Hiller, "Hábitat preferido de Lepidópteras", Comunicación persona (10 de agosto 2017).

[25] B. Louman, J. Valerio y W. Jiménez, "Bases ecológicas. Silvicultura de bosques latifoliados húmedos con énfasis en América Central”. Serie Técnica. Manual Técnico, CATIE, Costa Rica no.135 , pp.19-78, 2001.

[26] J. Vandermeer, N. Zamora, K. Yih y D. Boucher, "Regeneración inicial en una selva tropical en la costa caribeña de Nicaragua después del huracán Juana", Revista Biología Tropical, vol. 38 no. 2B, pp. 347-359, 1990.

[27] K. McGinley, "El manejo del bosque natural tropical y su impacto en la diversidad de la fauna. Una visión general integrada", Revista Forestal Centroamericana, vol. 32, pp. 34-38, 2000.

[28] J. Valerio, E.A. Esquivel y C. Salas, "Sistema de Parcelas Permanentes y análisis de la dinámica del bosque natural". Informe Final: Proyecto Manejo Integrado del Bosque. ITCR. Costa Rica, p 28, 1997.

[29] J. Araya, "Efectos del Huracán Otto sobre la estructura y composición florística de un bosque primario en Bocatapada de Pital, San Carlos, Costa Rica", Tesis, Instituto Tecnológico de Costa Rica, p. 42, 2017.

[30] F. H. Rosmidi, M.A. Zahidin, A. Adanan, A. Azizah, E. Pesiu y M.T. Abdullah, "Checklist of Butterflies in Pulau Perhentian and Pulau Bidong, Terengganu", Journal of Sustainability Science and Management, vol. 12, no. 1, pp. 40-48, 2017.

[31] P. J. De Vries, "Stratification of fruit-feeding nymphalid butterflies in a Costa Rican rainforest" Journal of Research on the Lepidoptera, vol. 26, no. 1-4, pp. 98-108, 1988.

[32] R. A. Raguso y J. Llorente-Bousquets, "The butterflies (Lepidoptera) of the Tuxtlas Mts., Veracruz, Mexico, revisited: species-richness and habitat disturbance", Journal of Research on the Lepidoptera, vol. 29 no. 1-2, pp-. 105-133,1990.

[33] K. Spitzer, J. Jaros, J. Havelka y J. Leps, "Effect of small-scale disturbance on butterfly communities of an Indochinese montane rainforest", Biological conservation, vol. 80, no. 1, pp. 9-15, 1997. 
[34] K. S. Brown y R. W. Hutchings, "Disturbance, fragmentation, and the dynamics of diversity in Amazonian forest butterflies". Tropical forest remnants: ecology, management, and conservation of fragmented communities. Chicago, The University of Chicago Press, pp. 632, 1997.

[35] L. A. Ospina-López, "Estructura de la comunidad de mariposas diurnas (Lepidoptera, Hesperioidea y Papilionoidea) en distintos tipos de hábitats en la cuenca del Río Lagunillas Tolima-Colombia, Tesis, Universidad Nacional de Colombia, Facultad de Ciencias, Bogotá DC, Colombia, 2014.

[36] V. D. Carmona y T. V. Carmona, "La Diversidad de los análisis de diversidad". [The Diversity of Diversity Analyses]. Bioma, vol. 14, pp. 20-28, 2013.

[37] T. E. Lovejoy, Jr. R. O. Bierregaard, A. B. Rylands, J. R. Malcom, C. E. Quintela, L. H. Harper, Jr. J. S. Brown, A. H. Powell, G. V. N. Powell, H. O. R. Schubart y M. B. Hays, "Edge and other effects of isolation on Amazon forest fragments", Soule, M. E. (Ed.), Conservation Biology: The Science of Scarcity and Diversity.Sipauer Associates, Sunderland, M A, pp. 257-285, 1986.

[38] J. K. Hill, K. C. Hamer, L. A. Lace y W. M. T. Banham, "Effects of selective logging on tropical forest butterflies on Buru, Indonesia", Journal of Applied Ecology, vol. 32, no. 4, pp. 754-760, 1995.

[39] R. K. Colwell, R. K. Estimate: statistical estimation of species richness and shared species from samples. Version 9.0. User's guide and application. Disponible en: http://purl. oclc. org/estimates, 2013.

[40] J. L. Mallet and J. T. Longino, "Hostplant records and descriptions of juvenile stages for two rare species of Eueides (Nymphalidae)", Journal of the Lepidopterists Society, vol. 36 no. 2, pp. 136-144, 1982.

[41] N. V. Sánchez, L. E. Vargas-Castro, A. Sánchez y M. Amador, "Riqueza y abundancia de mariposas diurnas, escarabajos coprófagos y plantas en cultivos orgánicos y convencionales de tres regiones de Costa Rica". UNED Research Journal, vol. 5, no. 2, pp. 249-259, 2013.

[42] H. Villarreal, M. Álvarez, S. Córdoba, F. Escobar, G. Fagua, F. Gast,... y A. M. Umaña. "Métodos para el análisis de datos: una aplicación para resultados provenientes de caracterizaciones de biodiversidad". Manual de Métodos Para el Desarrollo de Inventarios de Biodiversidad. Instituto de Investigación de Recursos Biológicos Alexander von Humboldt, Bogotá, Colombia, pp. 185-226, 2006.

\section{Este artículo debe citarse como:}

Salas-Garita, C; Jones-Román, G. (2019). Manejo forestal sostenible del bosque y monitoreo ecológico en dos bosques muy húmedos tropicales de Zona Norte de Costa Rica . Revista Forestal Mesoamericana Kurú, 16(39), 10-22. doi. 10.18845/ rfmk.v16i39.4408 